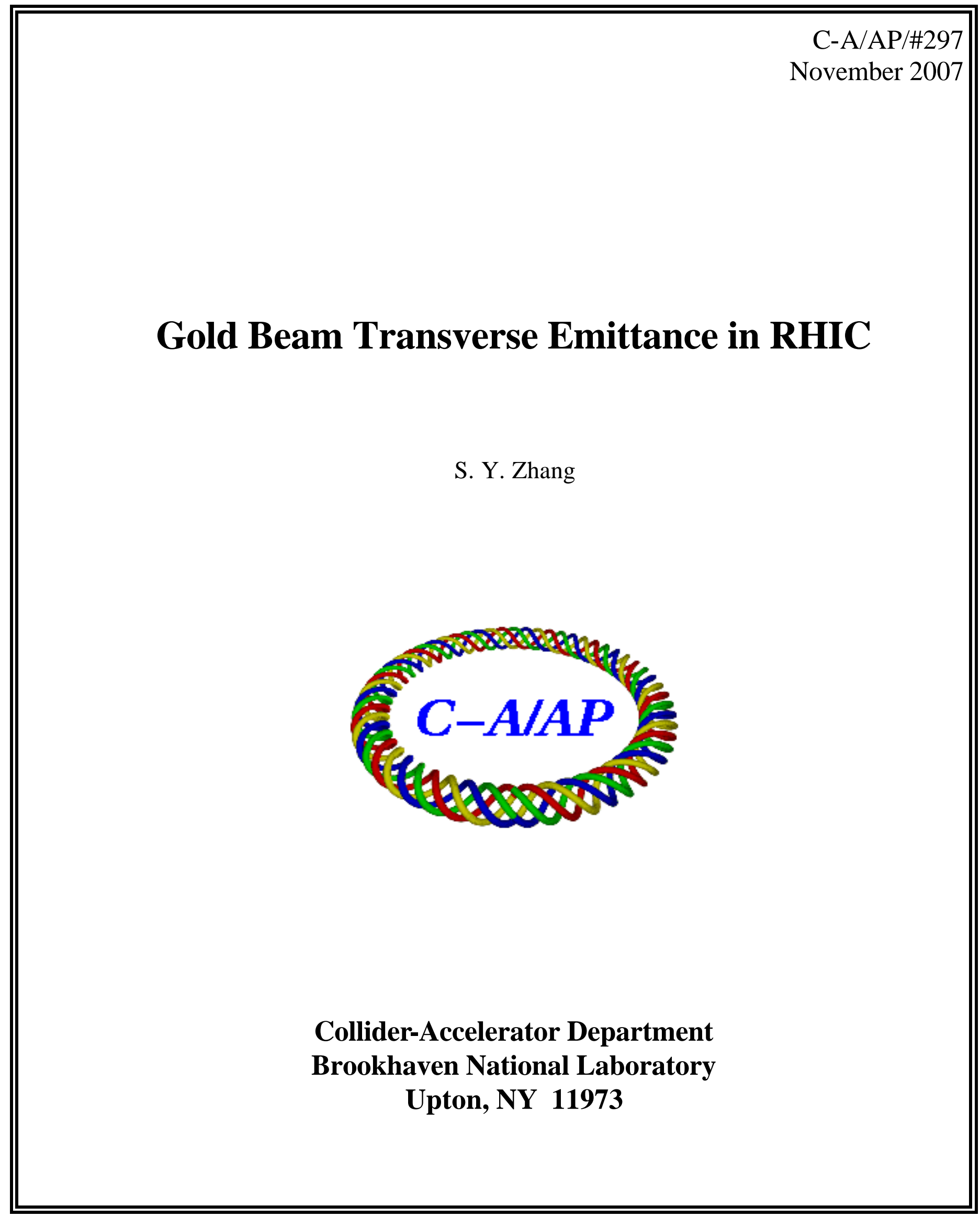




\title{
Gold Beam Transverse Emittance in RHIC
}

\author{
S.Y. Zhang
}

\begin{abstract}
The gold beam transverse emittance in Run 2007 was about $20 \%$ larger than in Run 2004. Since the gold beam transverse emittance was a dominant factor in terms of luminosity in deuteron-gold run 2003 , it is of interest in deuteron-gold run 2008. Some studies are proposed.
\end{abstract}

\section{Introduction}

In gold run 2007 (Au07), the beam emittance at early store in RHIC, measured using coincident collision rates, was about 20\% larger than in Run 2004 (Au04). With the same bunch intensity and similar bunch length, the emittance growth rate in store is quite different in the two runs, i.e., in Au07 it is much slower than in Au04. Since the dominant emittance growth mechanism in store is the intra-beam scattering (IBS) for gold beam, this suggests that the beam emittance in $\mathrm{Au} 07$ is indeed larger than Au04. As a result, typical Au04 fills with 45 bunches and the bunch intensity of $1.1 \times 10^{9}$ gold ions have peak collision rate of $15 \mathrm{kHz}$ for PHENIX. Typical Au07 fills with 103 bunches and same bunch intensity have $34 k H z$. The effect of smaller $\beta^{*}$ in Au07 is practically cancelled by the larger emittance. The root cause is not clear at the moment, but the larger emittance is suspected due to the injectors.

For deuteron-gold run 2003 (dAu03), it is found that the gold beam emittance, compared with the deuteron, is dominant in terms of the luminosity. The beam emittance, derived using the coincident collision rate at early store, has no dependence on the deuteron bunch intensity up to $1.1 \times 10^{11}$, but there is some dependence for the gold bunch intensity larger than $0.6 \times 10^{9}$. The latter is in agreement with the AGS to RHIC transfer line (ATR) measurement during the Au07 run. 
Based on the dAu03 experience, and considering the further increase of the gold beam transverse emittance in $\mathrm{Au07}$, the gold emittance is expected to affect the luminosity for deuteron-gold run 2008 (dAu08). It is of interest to have a better understanding for the large gold beam emittance. Studies seeking for possible gold beam emittance reduction might be pursued.

\section{Transverse emittance in $\mathrm{Au}-\mathrm{Au}$ runs 2004 and 2007}

In RHIC, the beam emittance can be evaluated using the PHENIX and STAR zero degree calorimeter (ZDC) coincident collision at the early store. The results are surprisingly more consistent than other measurements, and hence useful. This way, some aspects of the emittance, such as the Blue or Yellow beams, horizontal or vertical, cannot be identified. This shortcoming is somewhat made up by using other devices, such the ionization profile monitor (IPM), polarimeter target, and vernier scan.

In specific, the beam emittance is taken at 0.5 hour after the beam transition, calculated from the PHENIX ZDC coincident rate for Au04 and Au07. The $\beta^{*}$ of 1 meter and 0.8 meter are used for Au04 and Au07, respectively.

The results are shown in Fig.1, where the normalized emittance is plotted against the bunch intensity. For both runs, the dependence of the emittance on bunch intensity can be observed. The floor (lower bound) emittance, which is achieved when the machine tuning is optimized, of Au07 is larger than Au04.

In evaluating the difference of the emittance, several factors have to be considered, such as the real $\beta^{*}$ at the collision, the ZDC detector calibration, etc. However, most of the uncertainties can be ruled out if one looks at the emittance growth rate in store.

The beam emittance growth in store is dominated by the IBS for the gold ions. Given the same lattice and energy, the IBS caused emittance growth rate depends mainly on the bunch density, which in turns depends on the bunch intensity, bunch length, and the transverse emittance.

The beam emittance growth rate per hour in store, from 0.5 hour to 3.5 hour after the transition, is plotted against the bunch intensity in Fig.2 for Au04 and Au07. The growth rate dependence on the bunch intensity is expected, which affects the bunch density after all. For same bunch intensity, 


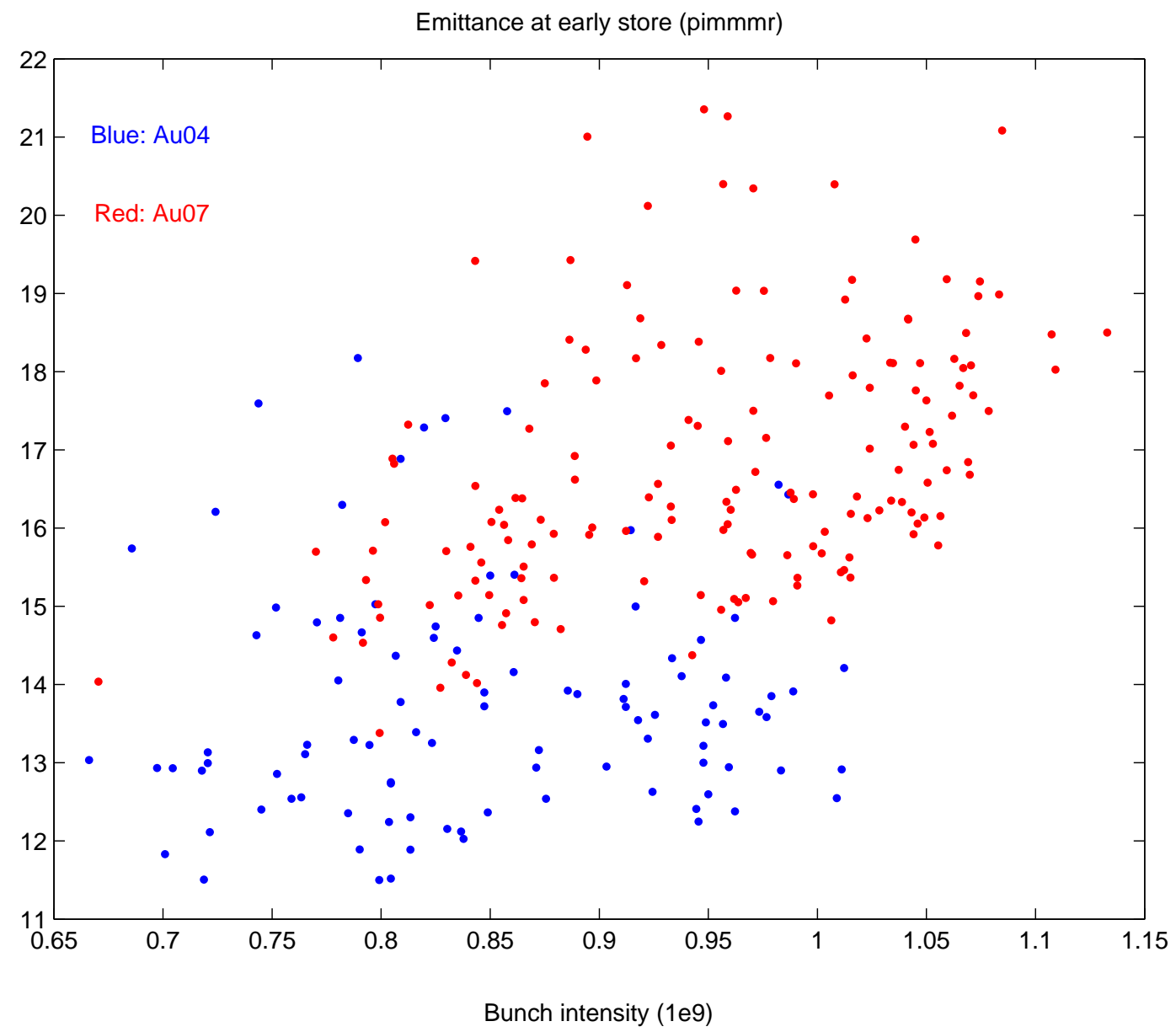

Figure 1: Normalized gold beam emittance vs. bunch intensity, in Au04 and Au07. The emittance is calculated using the PHENIX ZDC coincident collision rate at early store, 0.5 hour after the transition. $\beta^{*}$ is $1 \mathrm{~m}$ and 0.8 $\mathrm{m}$ for $\mathrm{Au} 04$ and Au07, respectively. 
the growth rate in Au07 is significantly smaller than Au04. Since the emittance calibration is little relevant here and the bunch lengths in two runs are very similar, this can only suggest that the emittance in Au07 is larger than $\mathrm{Au} 04$.

In Fig.3, the emittance growth rate is plotted against the bunch density in a unit of $10^{-6} /(\pi \mu m \cdot n s)$. As expected, the difference between the two runs is disappeared.

\section{Au beam emittance in d-Au runs 2003 and 2008}

In deuteron-gold runs, it is important to identify which beam has larger transverse emittance, and hence dominant in terms of luminosity. This is attempted for dAu03 by identifying the dependence of the emittance at early store with the deuteron and gold bunch intensities, respectively.

In Fig.4, it is shown that the beam emittance derived from the coincident collision rate has some dependence on the gold bunch intensity, but not the deuteron. For highest deuteron bunch intensities, two fills $(3236,3238)$ have the emittance larger than $10 \pi \mu m$, but these two also have high gold bunch intensities, about $0.6 \times 10^{9}$ gold ions. Fill 3154 with small emittance, about 7 $\pi \mu m$, has high deuteron bunch intensity but also a low gold bunch intensity, a little more than $0.4 \times 10^{9}$ gold ions. This shows two possibilities. One is that the deuteron transverse emittance is insensitive to the bunch intensity up to $1.1 \times 10^{11}$, and another is that the deuteron emittance is smaller than the gold. Either way, the gold beam emittance is dominant in dAu03 in terms of luminosity improvement.

One may find that in Fig.4, there are many fills with very large emittances. This happened at early run, with the deuteron beam transition instability not corrected. These fills are easily identified with low bunch intensities.

Compared with the beam emittance at early store in Au04 and Au07, typically at $13 \pi \mu m$ and $16 \pi \mu m$, as shown in Fig.1, the emittance in dAu03 is much smaller, typically at $10 \pi \mu \mathrm{m}$. This may be because that the gold bunch intensity was low, no more than $0.7 \times 10^{9}$ gold ions in dAu03. In Fig.5, putting the emittance in dAu03 and Au04 together against the gold bunch intensity, some alignment of the emittance vs. bunch intensity can be observed. This may serve as another argument that the gold beam emittance 


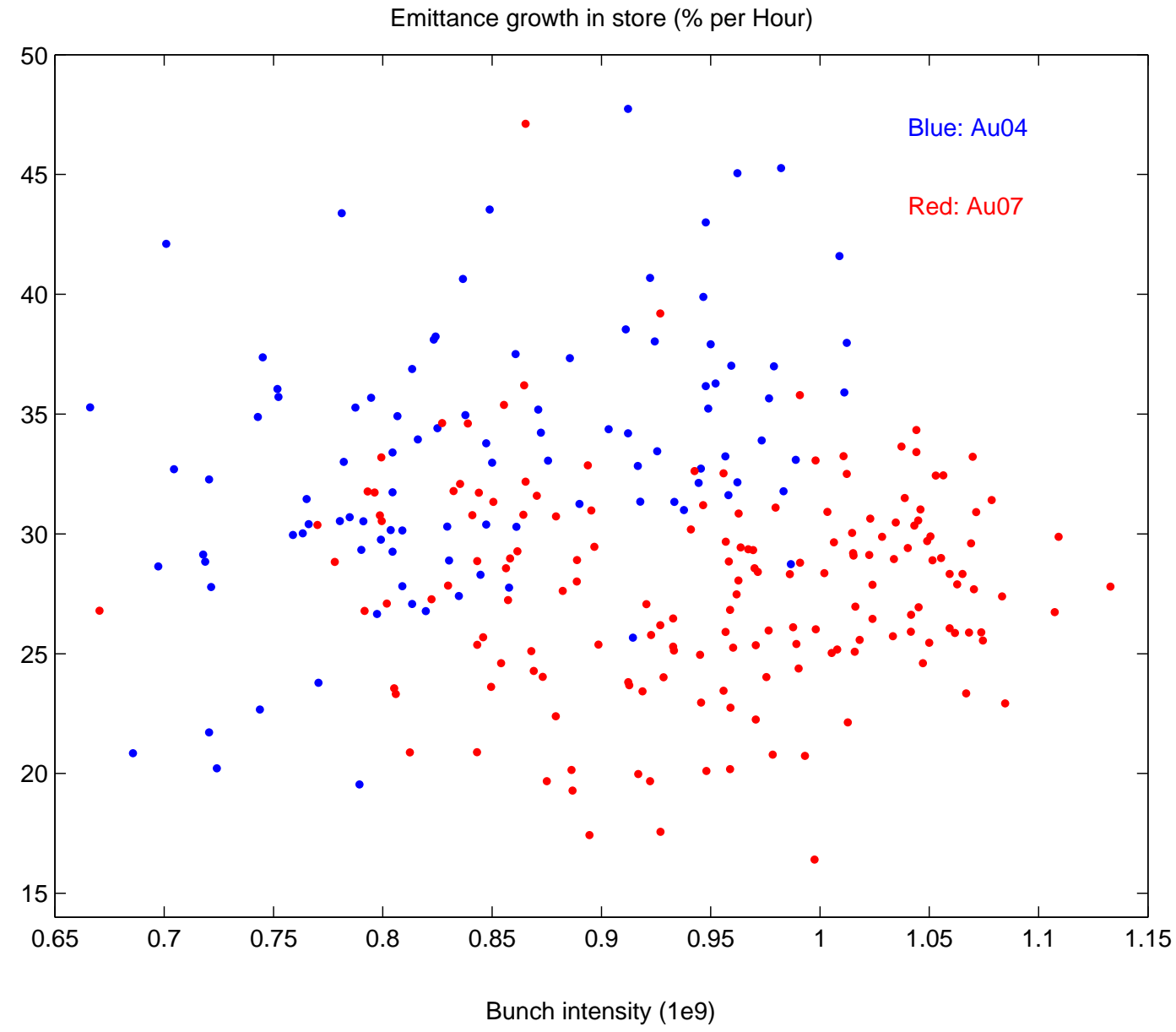

Figure 2: Gold beam emittance growth in store, averaged from 0.5 hour to 3.5 hour after the transition, in percentage per hour, against the bunch intensity. For same bunch intensity, the emittance growth rate in $\mathrm{Au07}$ is significantly smaller than Au04. 
Emittance growth in store (\% per Hour)

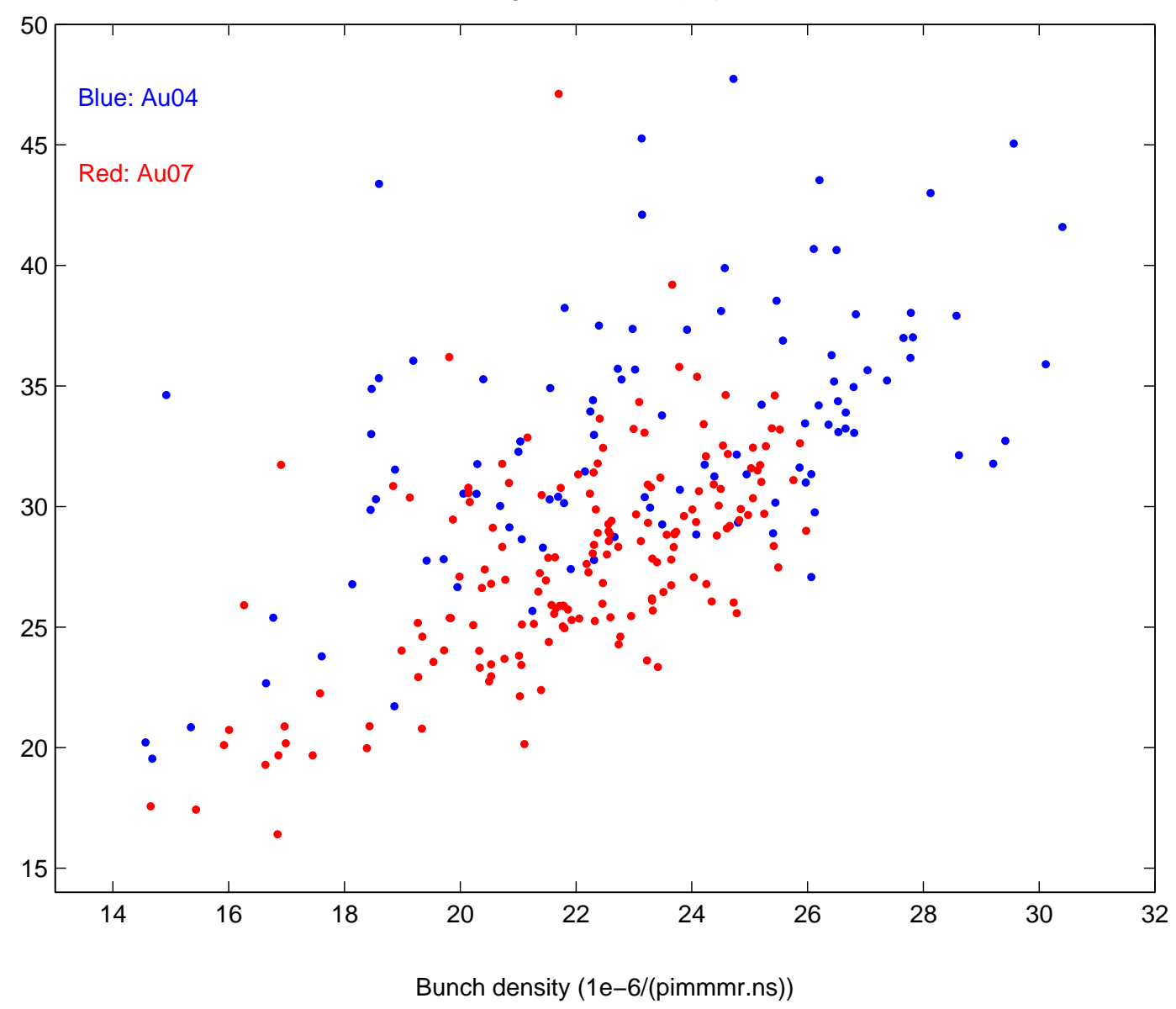

Figure 3: Gold beam emittance growth in store against the bunch density for $\mathrm{Au} 04$ and $\mathrm{Au} 07$. The bunch density is represented by the bunch intensity divided by the transverse emittance and bunch length. The emittance growth rate can be aligned by bunch densities, demonstrating that the dominant emittance growth mechanism is the IBS. 
Emittance at early store (pimmmr)
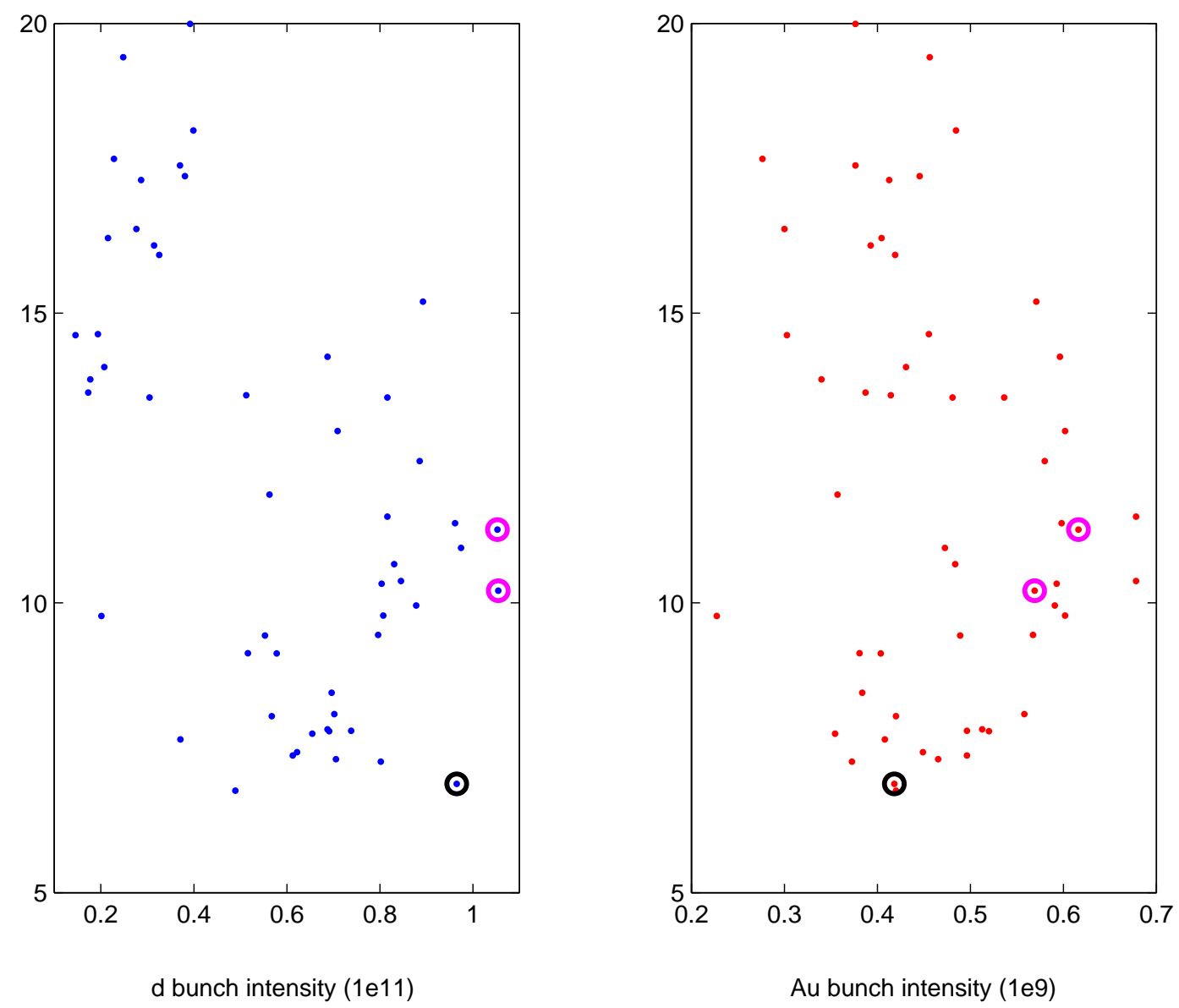

Figure 4: The beam emittance at early store in dAu03 vs. deuteron and gold bunch intensities. The emittance is measured using the coincident collision rate, and therefore it is indiscernible for deuteron or gold beams. Two fills with magenta circle are 3236 and 3238 , the one with black circle is 3154 . No dependence of the emittance on deuteron bunch intensity, but there is some dependence on gold. 
is important in $\mathrm{dAu}$ runs.

During the $\mathrm{Au} 07$ run, there was a systematic beam emittance measurement at the ATR transfer line [1]. The dependence of the emittance on the bunch intensity is shown in Fig.6, where the beam emittance at RHIC early store in Au07 is also shown for comparison.

There are several comments.

- To compare with the emittance at early store, the bunch intensity in ATR measurement is scaled by assuming $85 \%$ transmission from the ATR to RHIC early store, which is 0.5 hour after the transition. The beam loss includes the RHIC injection, ramping, and at early collision.

- The emittance at early store is about 2 to $3 \pi \mu m$ larger than the vertical emittance measured at the ATR. Consider the emittance growth in the ramping and the early collision, this seems reasonable.

- The vertical emittance in the ATR measurement appears less sporadic than the horizontal. This may because that the AGS is usually more affected by the momentum aperture limit. During the operations, most machine tuning at the AGS involves a lot more in the horizontal than vertical.

- One may notice that the ATR vertical emittance measured in Au07 looks very close to the RHIC beam emittance at early in dAu03 and $\mathrm{Au04}$. If the 2 to $3 \pi \mu m$ emittance growth during the RHIC ramping and early collision can be taken, then this shows that the gold beam emittance in $\mathrm{Au} 04$ is indeed smaller than $\mathrm{Au} 07$ by that amount.

- The ATR emittance measurement during Au04 is much less systematic. Some available data, however, show smaller emittance in both vertical and horizontal compared with $\mathrm{Au07}$ at the similar bunch intensities.

\section{Possible studies}

Two possible studies toward the improvement of the gold beam transverse emittance in dAu08 can be considered.

The first is focused on the Booster injection, which defines the gold beam emittance in the Booster [2]. Adjust injection turns and possibly modify the injection bumps may achieve improvement in the gold emittance. For given 


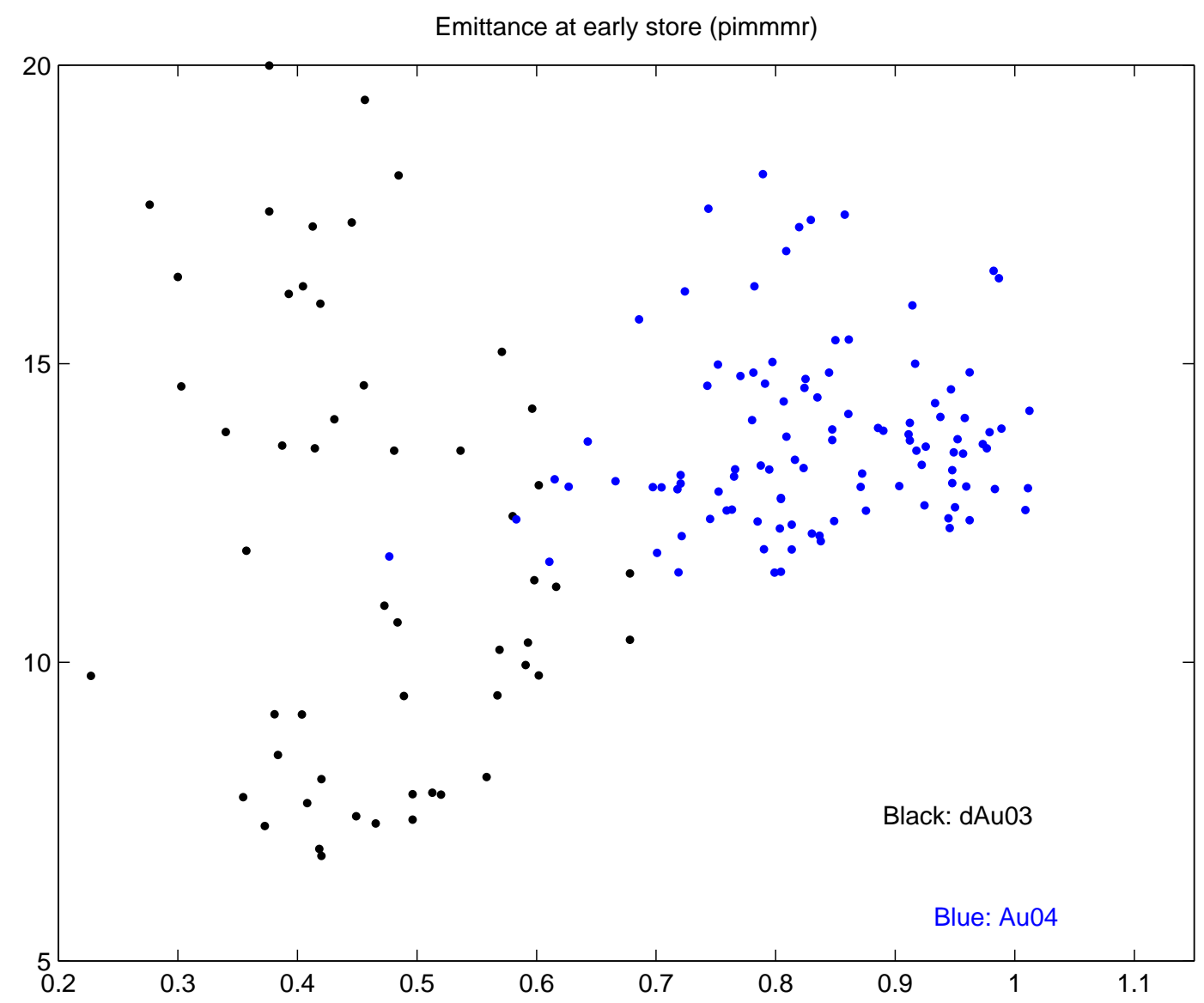

Figure 5: Beam emittance at early store, measured using the coincident collision rate, for $\mathrm{dAu} 03$ and Au04. The alignment of the emittance increase with the gold bunch intensity can be observed. 


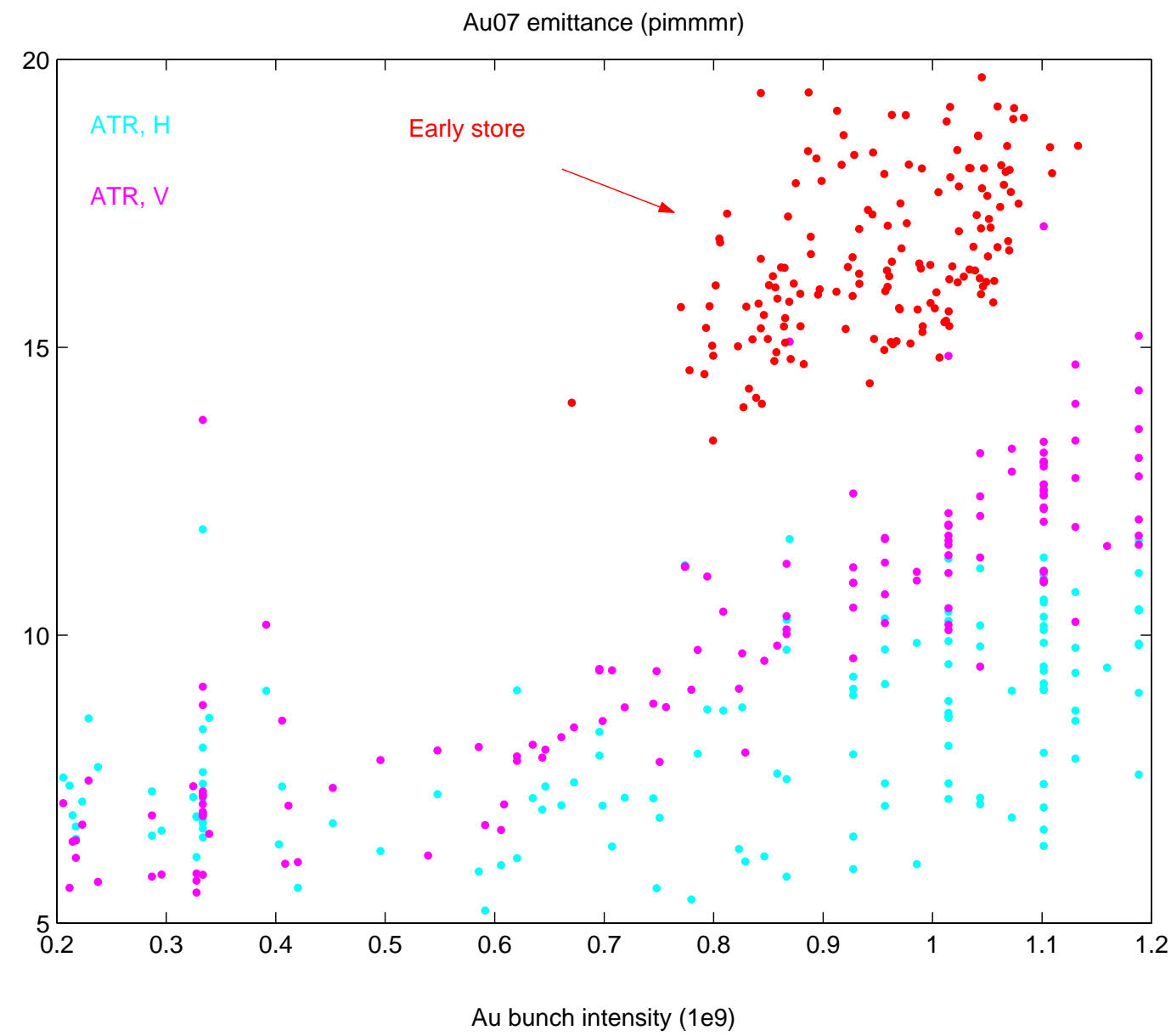

Figure 6: Beam emittance at the ATR transfer line, measured using the uline flags, against the intensity of the average of the transformers of uxf1, uxf3, and wxf1. The RHIC emittance at early store is also shown. To compare the differences, the ATR beam intensity is scaled back assuming $85 \%$ transmission from the ATR to RHIC early store. 
gold bunch intensity required at RHIC, the limit at the Tandem can be a problem.

The second study can be using the Booster vertical scraping to reduce the transverse emittance.

The Booster vertical scraping was applied in the polarized proton run 2006. First, the injector can provide higher bunch intensity the RHIC needed. Second, the transverse emittance growth with the proton bunch intensity is less significant, and third, the Booster vertical scraping generated smaller transverse emittance is beneficial for the polarization conservation in the AGS.

For gold beams, currently the injector chain may produce more than $1.5 \times 10^{9}$ gold ions per bunch for RHIC, which is more than needed. Compared with the protons, however, the gold beam transverse emittance has a significant enlargement at higher bunch intensities. For example, from the bunch intensity $0.6 \times 10^{9}$ gold ions to $1.2 \times 10^{9}$, the vertical emittance measured at ATR is almost doubled, as shown in Fig.6. This poses a question that if a pure gain is achievable by the Booster vertical scraping. As shown in Fig.6, there is some data with small horizontal emittance at ATR for high bunch intensities. If that is due to the horizontal scraping at the Booster and/or AGS, then it might indicate the possibility of the emittance reductions with scrapings.

\section{Acknowledgment}

The author would like to thank L. Ahrens, K. Brown, V. Ptitsyn, N. Tsoupas, and K. Zeno for helpful discussion.

\section{References}

[1] N. Tsoupas, private communications.

[2] K. Zeno, private communications. 